\title{
Influence of the Surface Viscosity on the Breakup of a Surfactant-Laden Drop
}

\author{
A. Ponce-Torres, ${ }^{1}$ J. M. Montanero, ${ }^{1}$ M. A. Herrada, ${ }^{2}$ E. J. Vega, ${ }^{1}$ and J. M. Vega ${ }^{3}$ \\ ${ }^{1}$ Depto. de Ingeniería Mecánica, Energética y de los Materiales and Instituto de Computación Científica Avanzada (ICCAEx), \\ Universidad de Extremadura, E-06006 Badajoz, Spain \\ ${ }^{2}$ Depto. de Mecánica de Fluidos e Ingeniería Aeroespacial, Universidad de Sevilla, E-41092 Sevilla, Spain \\ ${ }^{3}$ E.T.S.I. Aeronáutica y del Espacio, Universidad Politécnica de Madrid, Plaza Cardenal Cisneros 3, 28040 Madrid, Spain
}

(Received 2 September 2016; revised manuscript received 4 November 2016; published 13 January 2017)

\begin{abstract}
We examine both theoretically and experimentally the breakup of a pendant drop loaded with an insoluble surfactant. The experiments show that a significant amount of surfactant is trapped in the resulting satellite droplet. This result contradicts previous theoretical predictions, where the effects of surface tension variation were limited to solutocapillarity and Marangoni stresses. We solve numerically the hydrodynamic equations, including not only those effects but also those of surface shear and dilatational viscosities. We show that surface viscosities play a critical role to explain the accumulation of surfactant in the satellite droplet.
\end{abstract}

DOI: 10.1103/PhysRevLett.118.024501

The use of surfactants possesses a number of applications in diverse technological fields like ink-jet printing, the food industry, and biotechnology. For instance, surfactants enhance the stability of emulsions and bubbles, reduce the surface tension to adjust wettability conditions, regulate the transport conditions across interfaces, and enable the encapsulation of cells and biomolecules in droplets (see, e.g., Ref. [1] and references therein). The presence of a surfactant monolayer significantly affects the dynamical response of capillary systems. In fact, the spatiotemporal scales characterizing the fluid-dynamical problem may be comparable with those of the surfactant transport, which gives rise to complex and interesting phenomena. Attention has been frequently paid to the influence of the Marangoni convection caused by a nonhomogeneous surfactant concentration over the interface. On the contrary, the role played by the surface viscous stresses [2] associated with the surfactant monolayer has been neglected in most studies for no apparent physically meaningful reason. While both surface diffusion and Marangoni convection opposes to surfactant concentration variations over the interface, surface viscous stresses reduce the surface velocity gradients. A natural question is whether this latter mechanism can be comparable to or even dominate Marangoni convection in the problems analyzed.

In this Letter, we will show how surface viscous stresses can fundamentally change the dynamics of surfactant-laden capillary systems. For this purpose, we have selected a paradigmatic phenomenon in surface tension-driven flows: the breakup of a pendant drop. Specifically, we will study, experimentally first and then theoretically, the influence of an insoluble surfactant monolayer on the free surface pinching and the subsequent formation of satellite droplets. This phenomenon is essentially independent of the specific configuration (pendant drop, liquid bridge, liquid lens, jet, ...) considered. Although we restrict ourselves to insoluble and Newtonian surfactants because the model is more tractable in this case, one can expect the main conclusions to be applicable to soluble surfactants with adsorption and desorption time scales much larger than the breakup time, and to non-Newtonian surface active agents as well.

During the free surface breakup, the liquid evacuates from the pinch-off region, and the resulting surface convection sweeps away the surfactant accumulated there. Several numerical studies [3-7] have predicted that surface diffusion and Marangoni convection fail to restore the initial homogeneous surfactant density, and the pinch-off dynamics eventually approach the universal self-similar solution for clean interfaces [8]. In addition, those two dissipative effects are not capable of retaining the surfactant in the satellite droplet formed after the breakup. As will be shown, our experimental results contradict these predictions, and can be explained when surface viscous stresses are accounted for in a simple way.

In our experiments, use was made of the insoluble surfactant $\mathrm{C}_{18} \mathrm{OH}$, which is well characterized and has been used in previous works [7]. The equilibrium surface tension of spread $\mathrm{C}_{18} \mathrm{OH}$ monolayers on water at $25^{\circ}$ is approximately given by the equation of state [7]

$$
\sigma=\sigma_{c}-\frac{1}{2}\left(\sigma_{c}-\sigma_{\min }\right)\left\{\tanh \left[\alpha\left(\Gamma-\Gamma_{\text {ref }}\right)\right]+1\right\},
$$

where $\sigma_{c}=72 \mathrm{mN} / \mathrm{m}, \sigma_{\min }=36 \mathrm{mN} / \mathrm{m}, \alpha=0.752 \mathrm{~m}^{2} /$ $\mu \mathrm{mol}, \Gamma$ is the surfactant surface density, and $\Gamma_{\text {ref }}=$ $6.46 \mu \mathrm{mol} / \mathrm{m}^{2}$ (Fig. 1, left).

A pendant drop of deionized water was formed by injecting this liquid across a vertical needle $3.4 \mathrm{~mm}$ in diameter. $\mathrm{A} \mathrm{C}_{18} \mathrm{OH} /$ hexane solution was gently deposited on the pendant drop surface with a micropipette. After 

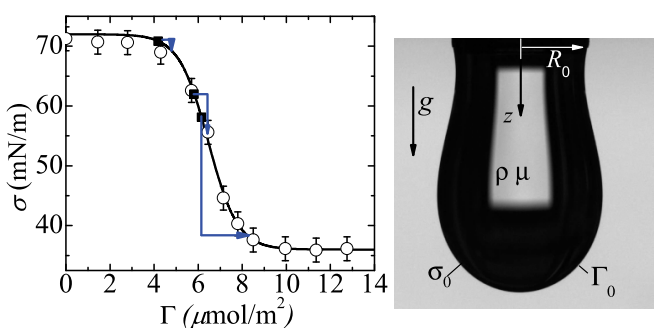

FIG. 1. (Left) Equilibrium surface tension of spread $\mathrm{C}_{18} \mathrm{OH}$ monolayers on water at $25^{\circ}$ [7]. The open symbols are the experimental data while the solid line is the fit (1) to them. The solid symbols correspond to the experimental realizations shown in Fig. 4, and the straight lines represent the surface tension drops during the satellite droplet formation in those experiments. (Right) Pendant drop at the maximum volume stability limit.

about $3 \mathrm{~min}$, the drop surface tension was measured with the drop shape tensiometer TIFA-AI [9]. If necessary, more $\mathrm{C}_{18} \mathrm{OH} /$ hexane solution was introduced onto the drop surface until the desired surface tension $\sigma_{1}$ was reached. The pendant drop free surface area $S_{1}$ was also measured from the drop image. The surfactant surface density $\Gamma_{1}$ was calculated from the isotherm (1). Then, the pendant drop was inflated by injecting more deionized water until the maximum volume stability limit was reached (Fig. 1, right). The pendant drop surface tension $\sigma_{0}$ and area $S_{0}$ were measured at that limit. For surface tensions sufficiently different from $72 \mathrm{mN} / \mathrm{m}^{2}$, the corresponding surfactant density $\Gamma_{0}$ was calculated from the equation of state (1). This calculation leads to considerable errors for $\sigma_{0} \simeq$ $72 \mathrm{mN} / \mathrm{m}$ due to the Plateau in this part of the curve $\sigma(\Gamma)$ (Fig. 1, left). In this case, $\Gamma_{0}$ was calculated from the surfactant mass conservation during the last drop inflation; i.e., $\Gamma_{0}=\Gamma_{1} S_{1} / S_{0}$. The result of the above experimental procedure is a pendant drop right at the maximum volume stability limit with known values of its surface tension $\sigma_{0}$ and surfactant density $\Gamma_{0}$ (Fig. 1, right).

The pendant drop broke up spontaneously (Fig. 2). In this process, a thin liquid thread forms between the upper

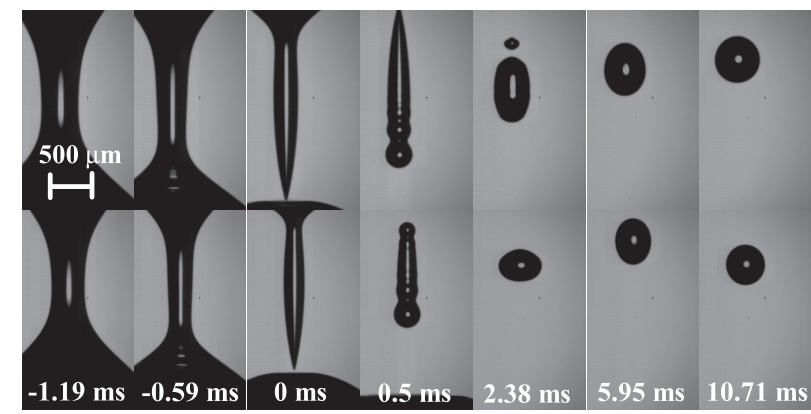

FIG. 2. Sequence of magnified images showing the formation of the satellite droplet. The upper (lower) images correspond to a clean (surfactant-laden, $\Gamma_{0}=6.38 \mu \mathrm{mol} / \mathrm{m}^{2}$ ) pendant drop. The instant $t=0$ is defined as that at which the free surface pinches for the first time. The corresponding videos can be found in the Supplemental Material [11]. and lower parent drops. Because of surface tension, the liquid thread contracts and oscillates until reaching a quasispherical shape. The last phase of these oscillations corresponds to the linear evolution of the axisymmetric mode $m=0$ and $l=2$ [10]. The frequency of this mode is approximately given by the equation $\omega=8\left(\rho d_{d}^{3} / \sigma_{d}\right)^{-1 / 2}$, where $\rho, d_{d}$, and $\sigma_{d}$ are the satellite droplet density, diameter, and surface tension, respectively. We measured the distance $d(t)$ between the north and south poles of the satellite droplet by processing images acquired at 84000 fps (Fig. 3). By fitting the function $d(t)=d_{d}+$ $a e^{-\gamma t} \cos (\omega t+\phi)$ to the experimental data, one obtains the diameter $d_{d}$, the frequency $\omega$, and therefore the surface tension $\sigma_{d}$ and the corresponding surfactant density $\Gamma_{d}\left(\sigma_{d}\right)$ given by the isotherm (1).

Figure 2 shows the breakup process of both a clean and a surfactant-laden pendant drop. Despite the relatively small value of $\Gamma_{0}$ in the latter case, these two processes are different qualitatively. The surfactant makes both the initial pendant drop and the central liquid thread smaller, and accelerates the free surface pinching at the upper drop. The satellite droplet ejects a tiny drop in the clean case, and its size is bigger than that produced by the surfactant-laden pendant drop. Figure 3, left shows the minimum free surface radius $R_{\min }$ as a function of the time to the pinching $\tau$. In all the cases, the asymptotic behavior $R_{\min } \propto \tau^{2 / 3}$ characterizing the inertiocapillary regime is reached $[8,12]$. The prefactor of that scaling law, and, therefore, the surface tension in the pinching point $[8,12]$, decreases as the initial surfactant concentration $\Gamma_{0}$ increases. This experimental result agrees with the theoretical predictions [5-7]. For $R_{\text {min }} \lesssim 30 \mu \mathrm{m}$, the curve slope slightly increases, a sign of the incipient viscous regime $[8,12]$. The so-called free surface overturning (free surface depression right before the
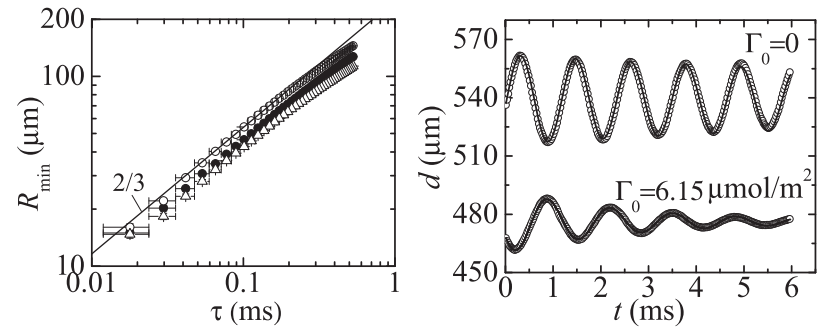

FIG. 3. (Left) Minimum free surface radius $R_{\min }$ as a function of the time to the pinching $\tau$ for $\Gamma_{0}=0$ (open circles), 6.38 (solid circles), and $7.82 \mu \mathrm{mol} / \mathrm{m}^{2}$ (triangles). (Right) Satellite droplet vertical size $d(t)$ after the breakup of a clean $\left(\Gamma_{0}=0\right.$ and $\left.\sigma_{0}=72.3 \mathrm{mN} / \mathrm{m}\right)$ and surfactant-laden $\left(\Gamma_{0}=6.15 \mu \mathrm{mol} / \mathrm{m}^{2}\right.$ and $\sigma_{0}=58.1 \mathrm{mN} / \mathrm{m}$ ) pendant drop. The lines are the fits of the function $d(t)=d_{d}+a e^{-\gamma t} \cos (\omega t+\phi)$ to the experimental data. These fits lead to $d_{d}=540$ and $476 \mu \mathrm{m}, \gamma=36.7$ and $62.6 \mathrm{~s}^{-1}$, and $\omega=5.44$ and $4.76 \mathrm{~s}^{-1}$ for the clean and surfactantladen drops, respectively. The corresponding surface tensions and surfactant densities are $\sigma_{d}=72.3$ and $38.3 \mathrm{mN} / \mathrm{m}$, and $\Gamma_{d}=0$ and $8.24 \mu \mathrm{mol} / \mathrm{m}^{2}$, respectively. 
pinch-off) disappears for $\Gamma_{0}>0$ even for the lowest concentration.

The comparison between the values of $d(t)$ for the clean and surfactant-laden drops (Fig. 3, right) clearly shows that a significant amount of surfactant is trapped in the satellite droplet. The surfactant monolayer increases the damping factor $\gamma$ and decreases the frequency $\omega$ of the oscillations. The surfactant density corresponding to the frequency in the surfactant-laden case is $\Gamma_{d}=8.24 \mu \mathrm{mol} / \mathrm{m}^{2}$, significantly larger than the initial value $\Gamma_{0}=6.15 \mu \mathrm{mol} / \mathrm{m}^{2}$, which contradicts all the theoretical predictions $[5-7,13,14]$. Therefore, there must be a physical mechanism not accounted for previously and responsible for such behavior. Here, we demonstrate that the shear and dilatational viscosities associated with the surfactant monolayer play a critical role in the process, and fundamentally change the drop dynamics.

In what follows, all the variables are made dimensionless with the needle radius $R_{0}$, the capillary time $t_{0}=$ $\left(\rho R_{0}^{3} / \sigma_{0}\right)^{1 / 2}$ ( $\rho$ is the bulk density), and the capillary pressure $\sigma_{0} / R_{0}$. The velocity $\mathbf{v}(\mathbf{r}, t)$ and reduced pressure $p(\mathbf{r}, t)$ fields are calculated from the continuity and NavierStokes equations $\boldsymbol{\nabla} \cdot \mathbf{v}=0$ and $\partial \mathbf{v} / \partial t+\mathbf{v} \cdot \boldsymbol{\nabla} \mathbf{v}=-\boldsymbol{\nabla} p+$ $\boldsymbol{\nabla} \cdot \mathbf{T}$, respectively, where $\mathbf{T}=C\left[\boldsymbol{\nabla v}+(\boldsymbol{\nabla v})^{T}\right]$ is the viscous stress tensor, and $C=\mu\left(\rho \sigma_{0} R_{0}\right)^{-1 / 2}$ is the volumetric Ohnesorge number ( $\mu$ is the bulk viscosity). These equations are integrated over the liquid domain of (dimensionless) volume $V$ considering the nonslip boundary condition at the solid surface, the anchorage condition at the needle edge, and the kinematic compatibility condition at the free surface. Neglecting the dynamic effects of the surrounding gas, the balance of normal stresses at the free surface yields $-p+B z+\mathbf{n} \cdot \mathbf{T} \cdot \mathbf{n}=\hat{\sigma} \kappa$, where $B=\rho g R_{0}^{2} / \sigma_{0}$ is the Bond number, $\mathbf{n}$ the unit outward normal vector, $\hat{\sigma} \equiv$ $\sigma / \sigma_{0}$ is the ratio of the local value $\sigma$ of the surface tension to its equilibrium value $\sigma_{0}$, and $\kappa$ is (twice) the mean curvature of the free surface. In addition, the balance of tangential stresses leads to $\mathbf{t} \cdot \mathbf{T} \cdot \mathbf{n}=\mathbf{t} \cdot \boldsymbol{\tau}^{S}$, where $\mathbf{t}$ is the unit vector tangential to the free surface meridians,

$$
\begin{aligned}
\boldsymbol{\tau}^{S}= & \boldsymbol{\nabla}^{S} \hat{\sigma}+\nabla^{S} \cdot\left\{C_{1}^{S}\left[\nabla^{S} \mathbf{v}^{S}+\left(\nabla^{S} \mathbf{v}^{S}\right)^{\top}\right]\right\} \\
& +\nabla^{S}\left[\left(C_{2}^{S}-C_{1}^{S}\right) \nabla^{S} \cdot \mathbf{v}^{S}\right],
\end{aligned}
$$

$\mathbf{v}^{S}(z, t)$ is the (two-dimensional) tangential velocity to the free surface [15], $\nabla^{S}$ the tangential intrinsic gradient along the free surface, and $C_{1,2}^{S}=\mu_{1,2}^{S}\left(\rho \sigma_{0} R_{0}^{3}\right)^{-1 / 2}$ are the superficial Ohnesorge numbers defined in terms of the surface shear and dilatational viscosities $\mu_{1}^{S}$ and $\mu_{2}^{S}$, respectively. These viscosities are expected to depend on the surfactant concentration. For the sake of simplicity, we assume the linear relationships $\mu_{1,2}^{S}=\mu_{1,2}^{S *} \Gamma$, where $\mu_{1,2}^{S *}$ are surfactant constants. Therefore, $C_{1,2}^{S}=\hat{C}_{1,2}^{S} \hat{\Gamma}$, where $\hat{C}_{1,2}^{S}=$ $\mu_{1,2}^{S *} \Gamma_{0}\left(\rho \sigma_{0} R_{0}^{3}\right)^{-1 / 2}$ are the superficial Ohnesorge numbers for the initial configuration, and $\hat{\Gamma} \equiv \Gamma / \Gamma_{0}$. To calculate this latter quantity, one must consider the equation governing surfactant transport on the free surface $\partial \hat{\Gamma} / \partial t+$ $\boldsymbol{\nabla}^{S} \cdot(\hat{\Gamma} \mathbf{v})=0$. In this equation, surface diffusion has been neglected because the surface Peclet numbers are typically in the range $10^{5}-10^{6}$ [16]. Finally, the Marangoni stress $\nabla^{S} \hat{\sigma}$ is evaluated by considering the isotherm (1). For $\hat{C}_{1}^{S}=\hat{C}_{2}^{S}=0$, one recovers the standard formulation where only solutocapillarity and Marangoni convection are taken into account $[5-7,13,14]$.

The above theoretical model is numerically solved by mapping the time-dependent liquid region onto a fixed numerical domain through a coordinate transformation. The hydrodynamic equations are spatially discretized with the Chebyshev spectral collocation technique, and an implicit time advancement is performed using second-order backward finite differences [17].

The problem has been formulated in terms of the nondimensional parameters $\left\{V, B, C, \hat{C}_{1}^{S}, \hat{C}_{2}^{S}\right\}$ and those characterizing the dimensionless form of the isotherm (1): $\left\{\sigma_{c} / \sigma_{0}, \sigma_{\min } / \sigma_{0}, \alpha \Gamma_{0}, \alpha \Gamma_{\text {ref }}\right\}$. In the experiments, the dimensional properties $\left\{g, R_{0}, \rho, \mu, \sigma_{c}, \sigma_{\min }, \alpha, \Gamma_{\text {ref }}\right\}$ were fixed, while the surfactant concentration $\Gamma_{0}$, and hence the surface tension $\sigma_{0}\left(\Gamma_{0}\right)$, was varied. The values of $\left\{V, B, C, \sigma_{c} / \sigma_{0}, \sigma_{\min } / \sigma_{0}, \alpha \Gamma_{0}\right\}$ were directly calculated from the experimental data, while the superficial Ohnesorge numbers $\hat{C}_{1}^{S}$ and $\hat{C}_{2}^{S}$ were adjusted for the simulation to reproduce the experimental satellite droplet diameter $d_{d}$ and surfactant concentration $\Gamma_{d}$ (Table I). Here, we consider four cases: clean free surface and three representative values of $\Gamma_{0}$ selected within the interval

TABLE I. Parameters characterizing the representative experimental realizations, as well as the diameter $d_{d}$ and variation $\hat{\Gamma}_{d}=\Gamma_{d} / \Gamma_{0}$ in the surfactant concentration of the satellite droplet. The superscripts "exp", "all", and "Mar" stand for the experimental measurements, the numerical results with all the effects (solutocapillarity, Marangoni convection, and surface viscosities), and the numerical results only with solutocapillarity and Marangoni convection, respectively.

\begin{tabular}{lcccccccccccccc}
\hline \hline$\Gamma_{0}\left(\mu \mathrm{mol} / \mathrm{m}^{2}\right)$ & $V$ & $B$ & $C \times 10^{2}$ & $\sigma_{c} / \sigma_{0}$ & $\sigma_{\min } / \sigma_{0}$ & $\alpha \Gamma_{0}$ & $\hat{C}_{1}^{S} \times 10^{2}$ & $\hat{C}_{2}^{S} \times 10^{2}$ & $d_{d}^{\text {exp }} / R_{0}$ & $d_{d}^{\text {all }} / R_{0}$ & $d_{d}^{\mathrm{Mar}} / R_{0}$ & $\hat{\Gamma}_{d}^{\mathrm{exp}}$ & $\hat{\Gamma}_{d}^{\text {all }}$ & $\hat{\Gamma}_{d}^{\mathrm{Mar}}$ \\
\hline 0 & 6.22 & 0.376 & 0.287 & 0.993 & 0.497 & 0 & 0 & 0 & 0.324 & 0.3276 & 0.3276 & 0 & 0 & 0 \\
4.19 & 6.01 & 0.385 & 0.291 & 1.02 & 0.508 & 3.151 & 0.53 & 1.4 & 0.320 & 0.3320 & 0.3168 & 1.04 & 1.030 & 0.1183 \\
5.82 & 5.29 & 0.440 & 0.311 & 1.16 & 0.581 & 4.376 & 0.48 & 0.3 & 0.316 & 0.3108 & 0.3000 & 1.09 & 1.103 & 0.4035 \\
6.15 & 5.02 & 0.469 & 0.321 & 1.24 & 0.620 & 4.624 & 0.6 & 0.7 & 0.284 & 0.2820 & 0.2708 & 1.34 & 1.330 & 0.5712 \\
\hline \hline
\end{tabular}




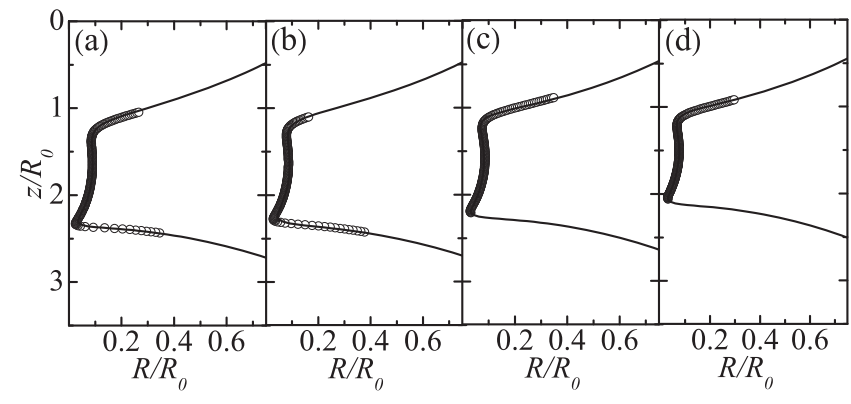

FIG. 4. Free surface radius $R(z)$ calculated numerically (solid lines) and measured experimentally (symbols) for $\Gamma_{0}=0$ (a), 4.19 (b), 5.82 (c), and $6.15 \mu \mathrm{mol} / \mathrm{m}^{2}$ (d). The time to the pinching was $\tau=77.3 \mu \mathrm{s}$ (a),(b) and $59.5 \mu \mathrm{s}$ (c),(d). The values of the parameters characterizing each case are displayed in Table I.

where $\sigma$ significantly depends on that parameter (Fig. 1, left).

Figure 4 shows the free surface position calculated numerically and measured experimentally at some instants before pinching. The remarkable agreement for the clean case shows the high accuracy of our numerical procedure. The experimental shapes are correctly reproduced by the simulations for the adjusted values of the superficial Ohnesorge numbers $\hat{C}_{1}^{S}$ and $\hat{C}_{2}^{S}$. We estimated the satellite droplet diameter $d_{d}$ from the volume of the thread connecting the two parent drops right before the free surface pinch-off. Table I shows the experimental and theoretical values of $d_{d}$, as well as those calculated for $\hat{C}_{1}^{S}=\hat{C}_{2}^{S}=0$, i.e., when only solutocapillarity and Marangoni convection are accounted for. Table I also displays the variation $\hat{\Gamma}_{d}=$ $\Gamma_{d} / \Gamma_{0}$ in the surfactant surface concentration of the satellite droplet. As can be observed, the inclusion of the surface viscosities does not considerably affect the satellite droplet diameter (this probably explains why the relevance of surface stresses in this phenomenon has not been previously detected). However, the role of these viscosities is essential to explain the increase of surfactant concentration in the satellite droplet. Essentially, surface viscosities oppose to velocity gradients along the free surface, which greatly hinders the outwards convection of surfactant in the central liquid thread of the pendant drop. As a consequence, a significant amount of surfactant is trapped in the satellite droplet, where the concentration becomes considerably larger than the average value. For $\Gamma_{0}=4.19 \mu \mathrm{mol} / \mathrm{m}^{2}$, solutocapillary effects and Marangoni stresses alone underestimate in 1 order of magnitude the surfactant surface concentration of the satellite droplet.

The surface viscosities in Table I are consistent with the literature values. Specifically, the surface shear viscosity value $2.3 \pm 0.1 \times 10^{-6} \mathrm{~Pa} \mathrm{~s} \mathrm{~m}$ has been reported [18] for a 1-octadecanol monolayer with a concentration very similar to the case $\Gamma_{0}=5.82 \mu \mathrm{mol} / \mathrm{m}^{2}$ in Table I. We obtained the value $2.6 \times 10^{-6} \mathrm{~Pa} \mathrm{~s} \mathrm{~m}$ for that case. Interestingly, surface viscous stresses alter considerably the surfactant transport over the free surface even when one considers surface viscosities in the lower limit of the range of values reported in the literature [19-23].

The present analysis shows the importance of surface viscosities in the dynamics of surfactant-laden capillary systems by considering the paradigmatic example of the pendant drop breakage. According to the previous numerical studies [3-7], Marangoni elasticity does not prevent surface convection from transporting surfactant away from the center of the pendant drop, and the resulting satellite droplet is cleaner than the parent droplets. Our experiments have clearly shown the opposite effect. The numerical simulations have explained this effect in terms of the surface viscosities. Therefore, the formulation of a completely predictive model (without adjustable variables) requires the knowledge of both the equation of state $\sigma(\Gamma)$ and the presumably nonlinear constitutive relationships $\mu_{1,2}^{S}=\mu_{1,2}^{S}(\Gamma)$. The conclusions derived from this analysis may be extrapolated to the breakup of liquid bridges, capillary tubes, liquid lenses and jets, charged not only with insoluble surfactants but also with soluble ones characterized by adsorption and desorption time scales much larger than the breakup time.

Partial support from the Ministerio de Economía y Competitividad and Gobierno de Extremadura (Spain) through Grants No. DPI2013-46485, No. TRA201345808-R, and No. GR10047, is gratefully acknowledged.

[1] S. L. Anna, Annu. Rev. Fluid Mech. 48, 285 (2016).

[2] L. E. Scriven, Chem. Eng. Sci. 12, 98 (1960).

[3] H. A. Stone and L. G. Leal, J. Fluid Mech. 220, 161 (1990).

[4] M.-L. E. Timmermans and J. R. Lister, J. Fluid Mech. 459, 289 (2002).

[5] R. V. Craster, O. K. Matar, and D. T. Papageorgiou, Phys. Fluids 14, 1364 (2002).

[6] P. T. McGough and O. A. Basaran, Phys. Rev. Lett. 96, 054502 (2006).

[7] Y.-C. Liao, E. I. Franses, and O. A. Basaran, Phys. Fluids 18, 022101 (2006).

[8] J. Eggers, Phys. Rev. Lett. 71, 3458 (1993).

[9] M. G. Cabezas, A. Bateni, J. M. Montanero, and A. W. Neumann, Langmuir 22, 10053 (2006).

[10] E. Becker, W. J. Hiller, and T. A. Kowalewski, J. Fluid Mech. 231, 189 (1991).

[11] See Supplemental Material at http://link.aps.org/ supplemental/10.1103/PhysRevLett.118.024501 for the videos corresponding to the experiments shown in Fig. 2.

[12] J. R. Castrejón-Pita, A. A. Castrejón-Pita, S. S. Thete, K. Sambath, I. M. Hutchings, J. Hinch, J. R. Lister, and O. A. Basaran, Proc. Natl. Acad. Sci. U.S.A. 112, 4582 (2015).

[13] B. Ambravaneswaran and O. A. Basaran, Phys. Fluids 11, 997 (1999).

[14] V. Dravid, S. Songsermpong, Z. Xue, C. M. Corvalan, and P. E. Sojka, Chem. Eng. Sci. 61, 3577 (2006). 
[15] J. M. Lopez and A. Hirsa, J. Colloid Interface Sci. 206, 231 (1998).

[16] Y.-M. Tricot, Surfactants: Static and Dynamic Surface Tension (Chapman and Hall, London, 1997), p. 100.

[17] M. A. Herrada and J. M. Montanero, J. Comput. Phys. 306, 137 (2016).

[18] T. Kato and M. Arai, J. Chem. Soc. Jpn. 12, 1594 (1991).
[19] N. Pilpel and R. P. Enever, Trans. Faraday Soc. 64, 231 (1968).

[20] H. Huhnerfuss, J. Colloid Interface Sci. 120, 281 (1987).

[21] L. Fourt and W. D. Harkins, J. Phys. Chem. 42, 897 (1937).

[22] K. S. Birdi, Self-Assembly Monolayer Structures of Lipids and Macromolecules at Interfaces (Springer, New York, 2002).

[23] H. Huhnerfuss, J. Colloid Interface Sci. 107, 84 (1985). 